\title{
Stratified analyses of genome wide association study data reveal haplotypes for a candidate gene on chromosome 2 (KIAA1211L) is associated with opioid use in patients of Arabian descent
}

Hiba Alblooshi ${ }^{1,2,3}$, Habiba Al Safar ${ }^{4,5}$, Ahmed El Kashef ${ }^{6}$, Hamad Al Ghaferi $^{6}$, Mansour Shawky ${ }^{6}$, Gary K. Hulse ${ }^{1,7}$ and Guan K. Tay ${ }^{1,4,5,7^{*}}$

\begin{abstract}
Background: Genome Wide Association Studies (GWAS) have been conducted to identify genes and pathways involved in development of opioid use disorder. This study extends the first GWAS of substance use disorder (SUD) patients from the United Arab Emirates (UAE) by stratifying the study group based on opioid use, which is the most common substance of use in this cohort.

Methods: The GWAS cohort consisted of 512 (262 case, 250 controls) male participants from the UAE. The samples were genotyped using the Illumina Omni5 Exome system. Data was stratified according to opioid use using PLINK. Haplotype analysis was conducted using Haploview 4.2.

Results: Two main associations were identified in this study. Firstly, two SNPs on chromosome 7 were associated with opioid use disorder, rs118129027 ( $p$-value $\left.=1.23 \times 10^{-8}\right)$ and rs74477937 $\left(p\right.$-value $\left.=1.48 \times 10^{-8}\right)$. This has been reported in Alblooshi et al. (Am J Med Genet B Neuropsychiatr Genet 180(1):68-79, 2019). Secondly, haplotypes on chromosome 2 which mapped to the KIAA1211L locus were identified in association with opioid use. Five SNPs in high linkage disequilibrium (LD) (rs2280142, rs6542837, rs12712037, rs10175560, rs1 1900524) were arranged into haplotypes. Two haplotypes GAGCG and AGTTA were associated with opioid use disorders ( $p$-value $3.26 \times 10^{-8}$ and $7.16 \times 10^{-7}$, respectively).

Conclusion: This is the first GWAS to identify candidate genes associated with opioid use disorder in participants from the UAE. The lack of other genetic data of Arabian descent opioid use patients has hindered replication of the findings. Nevertheless, the outcomes implicate new pathways in opioid use disorder that requires further research to assess the role of the identified genes in the development of opioid use disorder.
\end{abstract}

Keywords: Opioid use disorder, GWAS, stratification, Haplotypes, KIAA1211L

\footnotetext{
* Correspondence: guantay@bigpond.com

'Division of Psychiatry, the University of Western Australia, Crawley, Western

Australia, Australia

${ }^{4}$ Center of Biotechnology, Khalifa University of Science and Technology, Abu

Dhabi, United Arab Emirates

Full list of author information is available at the end of the article
}

(c) The Author(s). 2020 Open Access This article is distributed under the terms of the Creative Commons Attribution 4.0 International License (http://creativecommons.org/licenses/by/4.0/), which permits unrestricted use, distribution, and reproduction in any medium, provided you give appropriate credit to the original author(s) and the source, provide a link to the Creative Commons license, and indicate if changes were made. The Creative Commons Public Domain Dedication waiver (http://creativecommons.org/publicdomain/zero/1.0/) applies to the data made available in this article, unless otherwise stated. 


\section{Background}

Epidemiological data from the 2010 Global Burden of Diseases Health Measurement Survey estimated that some 15.5 Million people across the globe were afflicted with opioid use disorder [1]. As a measure of the chronic nature of the problem, the use of opioids significantly rose in the United States of America (USA) to epidemic proportions with a dramatic increase of $78 \%$ in 2013 [2]. Opioids of choice included heroin, as well as the substances morphine, fentanyl, oxycodone and tramadol which are widely used as prescribed analgesics for surgery [3]. The rise in the use of prescribed opioids for non-medical purposes has been reviewed in many countries [4]. The USA is considered the epicentre of the world's prescription drugs problem, where a 10 to 14 fold increase in prescription medication has been estimated $[5,6]$. The consumption of prescription medication for non-medical use resulting in harmful effects in 2010, was highest in the USA at 47,809 (consumption level per capita), followed by Canada $(26,380)$, the United Kingdom (UK) $(10,297)$ and Australia $(12,654)$ [7]. In addition, there was a massive increase of $430 \%$ in the number of treatment admission for prescription medication misuse between 1999 to 2009 in the USA [6]. Even though, there been as a dramatic increase in the prescription medication misuse in Europe, it is not as prevalent in the UK in comparison to the USA $[8,9]$. This is due to the strict regulation and legislations that control the use of prescription medication in the UK through the implementation of an electronic prescription system [4]. However, around $30 \%$ of patients with prescribed medication in the UK tend to sell or swap their allocation with other medications that contribute to the problem [10]. In North Africa and Middle East region, an estimated 1.37 million patients reported the use of opioids in 2010 [1]. In the first retrospective study of substance use disorder (SUD) in the United Arab Emirates (UAE) from 2002 to 2011, opioids (heroin $=16.3 \%$ ) were the second most common substance of use [11].

This pattern of prescription opioid use in the young (below the age of 30 years old) users from the UAE was recently described in Alblooshi et al (2016) [12]. The use of prescription opioids such as Tramadol rose by an estimated $67.2 \%$ in users below 30 years of age [12, 13]. This cohort was used in this study.

The vulnerability to substance use and treatment responses is partly affected by genetic factors [14-16] . The Identification of genes that contribute to the development of the disease can improve treatment outcomes of SUD. Opioid receptors (OPRM1, OPRD1 and OPRK1) have been extensively studied in addiction due to their involvement in the reward pathways. The $\mu$-opioid receptor (OPRM1) has been the main focus in opioid use disorder. Various Genome Wide Association Studies
(GWAS) [17-19] have looked into the genetic factors contributing to opioid use disorder. However, inconsistent genetic associations have been reported, suggesting that there are other systems involved in the pathogenesis of the opioid use disorder [20]. In the first GWAS study by Gelenter et al (2014) [17] the KCNG2 (rs62103177) gene on chromosome 18 was implicated. The association was mapped to a Calcium and Potassium pathway, a novel risk pathway that provided a new direction for therapeutic and preventative strategies [17]. Nelson et al (2015) [21] subsequently reported an association of the cornichon family AMPA receptor auxiliary protein 3 gene $(\mathrm{CNIH} 3)$ with heroin use disorder. This finding implicated the involvement of the glutamate system in the pathophysiology of opioid use disorder. To date, a number of genes, including LOC647946, FAM53B, CRYGS which encode proteins involved in different biochemical pathways have been reported. Presently, no definitive mechanism has been uncovered to explain the underlining pathophysiology of the opioid use disorder.

In many polygenic diseases, ethnic specific genetic variations have been described. In opioid use disorder, various GWA studies have been performed and reported in European American, African American [17, 22] and Australian population [21]. However, none have been performed on an Arab population. In this study, the first GWAS of SUD in a population of Arabian descent was conducted. The subjects were opioid users as this substance class was the most common substance of use at $80.4 \%$ [12] of the cohort studied. Three novel variants on chromosome 7 were identified and discussed in Alblooshi et al [23]. In this report, associations with haplotypes on chromosome 2 around the KIAA1211L locus are presented.

\section{Methods \\ Participants}

The GWAS discovery samples consisted of 262 male participants from the UAE. Cases included 250 male patients from the UAE National Rehabilitation Centre (NRC). All cases were diagnosed with SUD based on Diagnostic and Statistical Manual-5 (DSM-5) criteria. However, cases were not assessed for other psychiatric disorders at the time of recruitment. Controls with no prior history of SUD were retrieved from the Emirates Family Registry (EFR) [24] as a control group. However, other diseases were included in the control group selection criteria such as diabetes, cardiovascular diseases, dyslipidaemia, etcetera. The details of the cohort have been previously summarised in Alblooshi, et al (2016) [12] including the demographic characteristic and the type of substances used. The cohort was stratified based according to the common substance of use, which was opioids. 
The study was conducted in accordance with standards set by the World Medical of Helsinki [25]. The ethics committee of the National Rehabilitation Centre (NRC) in Abu Dhabi, UAE reviewed and approved the study. Reciprocal approvals were received from the ethics committee of the University of Western Australia (RA/4/1/6715). Only participants who signed written informed consent were studied.

\section{Genotyping and quality control}

Saliva samples were collected using the Oragene saliva kit (DNA Genoteck, Ottawa, Ontario, Canada). Genomic DNA was extracted using laboratory protocol for manual extraction as recommended by Genoteck. Each sample was processed for quantification using standard gel electrophoresis and Tecan NanoQuant Plate ${ }^{\mathrm{TM}}$ (Infinite 200 Pro) (Tecan Group, Männedorf, Switzerland). After extracting genomic DNA, the samples were genotyped using the Illumina Omni5 Exome (Illumina, San Diego, California), which contained 4.6 million Single Nucleotide Polymorphism (SNPs). Standard protocols as recommended by Illumina were used for hybridizing samples on the chip and scanning on the Illumina HiScan platform.

Quality Control (QC) step was applied on markers and individuals using PLINK [26]. The markers were filtered based on a genotype call rate $<99.6 \%$; Minor Allele Frequency $(\mathrm{MAF})<0.05$; significant deviation from Hardy Weinberg Equilibrium $\left(p\right.$-value $<10^{-6}$ ) and large difference $(p$-value $<0.00001)$ in missing data rate between cases and controls. The individuals were removed from the analysis where there was substantial missing data (> $90 \%$ ); when whole genome heterozygosity was greater than three standard deviation from the mean; estimated proportion of "identity by descent" (IBD) sharing with another sample $>0.1$, or gender discordant (based on $\mathrm{X}$ chromosomal heterozygosity) $>0.2$. Multi-dimensional scaling (MDS) was carried out to identify population outliers. After filtering for individuals, 199 cases with opioid use disorder and 262 controls were retained final analysis.

\section{Statistical analysis}

For the data stratified according to opioid use disorder, a total of 1,879,623 SNPs and 452 (Case:199 and Control: 253) passed QC. These were included in the study analysis. The GWAS association test was preformed using Factored Spectrally Transformed Linear Mixed Model "FaST-LMM" [27]. Quantile-Quantile (QQ) and Manhattan plot were illustrated using $R$ statistical package ( $R$ core team, Vienna, Austria). The GWAS significant level was set at $5.00 \times 10^{-8}$ [28]. The Regional Manhattan plot showing the SNP positions of interest were plotted using zoom locus webserver [29]. PLINK [26] was used for stratification of common substance of use. Haploview 4.2 [30] was used for haplotype and linkage disequilibrium (LD) analyses.

\section{Results}

In this report, analysis from a GWA study of SUD patients using opioids is presented. Fig. 1 shows the stratified GWAS Manhattan plot for opioid use disorder. In the analysis, two SNPs reached significant association with $p$-values of $1.23 \times 10^{-8}$ and $1.48 \times 10^{-8}$ for rs118129027 and rs74477937 respectively. These SNPs were localized to chromosome 7 . Another SNP rs78707086 on chromosome 7 failed to reach the set GWAS significance level $\left(p\right.$-value $\left.=5.00 \times 10^{-8}\right)$ but was suggestive of an association. Nevertheless, this third SNP was in LD with rs118129027 and rs74477937. The relevance of these three SNPs in SUD has been reported elsewhere [23].

A second association is described in this paper. Between the GWAS level of significance $\left(5.00 \times 10^{-8}\right)$ and the suggestive level $\left(1.00 \times 10^{-5}\right)$, seven SNPs on chromosome 2 formed a cluster with five of these SNPs in LD (Fig. 1). This suggested the possibility of candidate locus on chromosome 2 associated with opioid use disorder.

The cluster of SNPs circled in Fig. 1 highlight the nucleotide positions that have risen above the suggestive GWAS confidence level. A regional manhattan plot (Fig. 2) was generated around the location of these SNPs, partly to assess Linkage Disequilibrium (LD) between the SNPs. The recombination rates are shown in centimorgans (cM) per megabase $(\mathrm{Mb})$. Each SNP is represented as a circle with the $y$-axis shown as $-\log _{10} p$-value. The SNP with the highest $p$-value $\left(\right.$ rs10175560 with a $p$-value $=2.24 \times 10^{-7}$ ) was selected as the index SNP (purple diamond in Fig. 2). Linkage disequilibrium (LD) values with other SNPs in the vicinity of this index were estimated in $\mathrm{r}^{2}$ values and shown in different colours. The LD correlation was estimated using the data from the 1000 Genome project [29]. The highest LD is shown in red were for SNPs with $r^{2}>0.08$. SNPs with no LD data were represented by grey circles. Genes within the vicinity of the SNP of interest are provided. In this figure, the suggestive SNPs mapped to a region coinciding with a locus known as KIAA1211L. In addition, Fig. 2 illustrated strong LD that suggested further haplotype analysis for an association and improved evidence for candidate genes.

The SNPs on chromosome 2 that were above the suggestive line of $1.00 \times 10^{-5}$ determined by this GWA study are shown in Fig. 3. The data was not adjusted for other psychiatric disorder; hence the association level might be altered. Five out of seven SNPs on chromosome 2 above this suggestive line were in LD (rs2280142, rs6542837, rs12712037, rs10175560, 


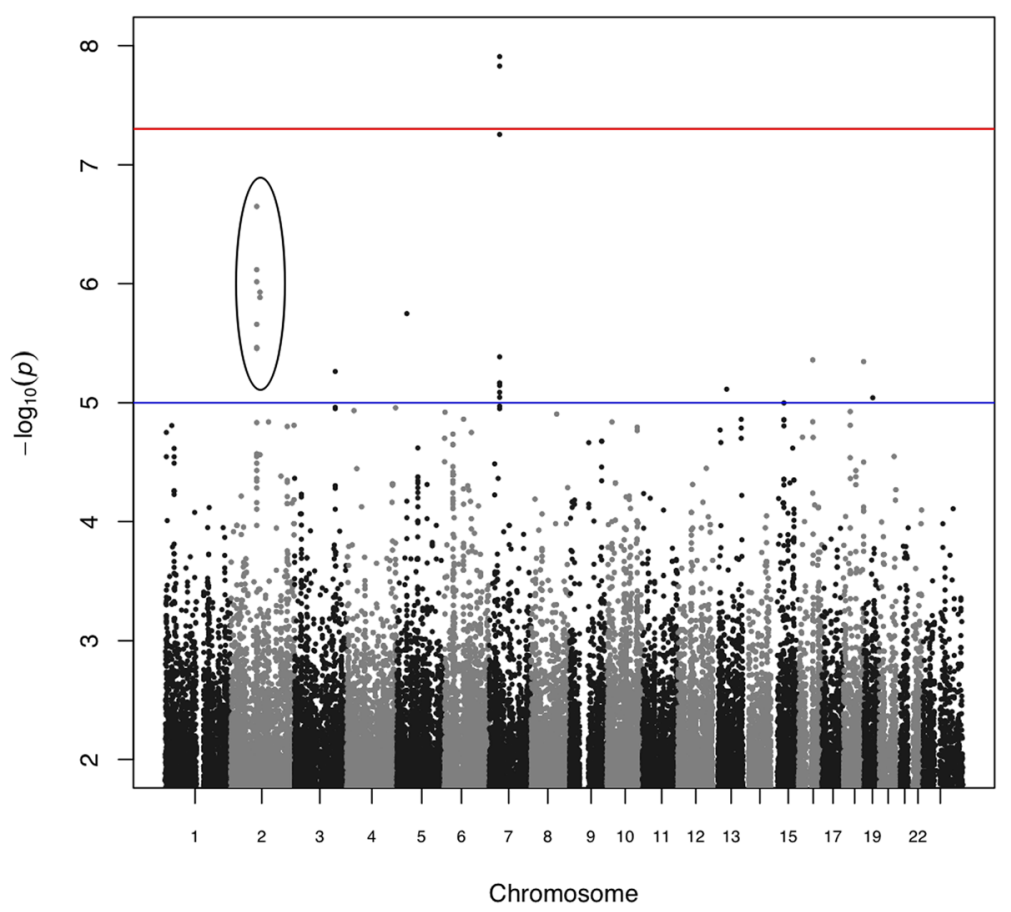

Fig. 1 Manhattan plot shows $p$-value for stratified opioid users versus normal controls using FaST-LMM analysis. The X-axis represents the SNP markers in each chromosome and Y-axis represent the negative logarithm of $P$-values. The highest $p$-values were observed for rs $118129027(p$ value $\left.=1.236 \times 10^{-8}\right)$ and rs $74477937\left(p\right.$-value $\left.=1.485 \times 10^{-8}\right)$ on chromosome 7 . The circle indicates a candidate haplotype that associated with opioid use disorder

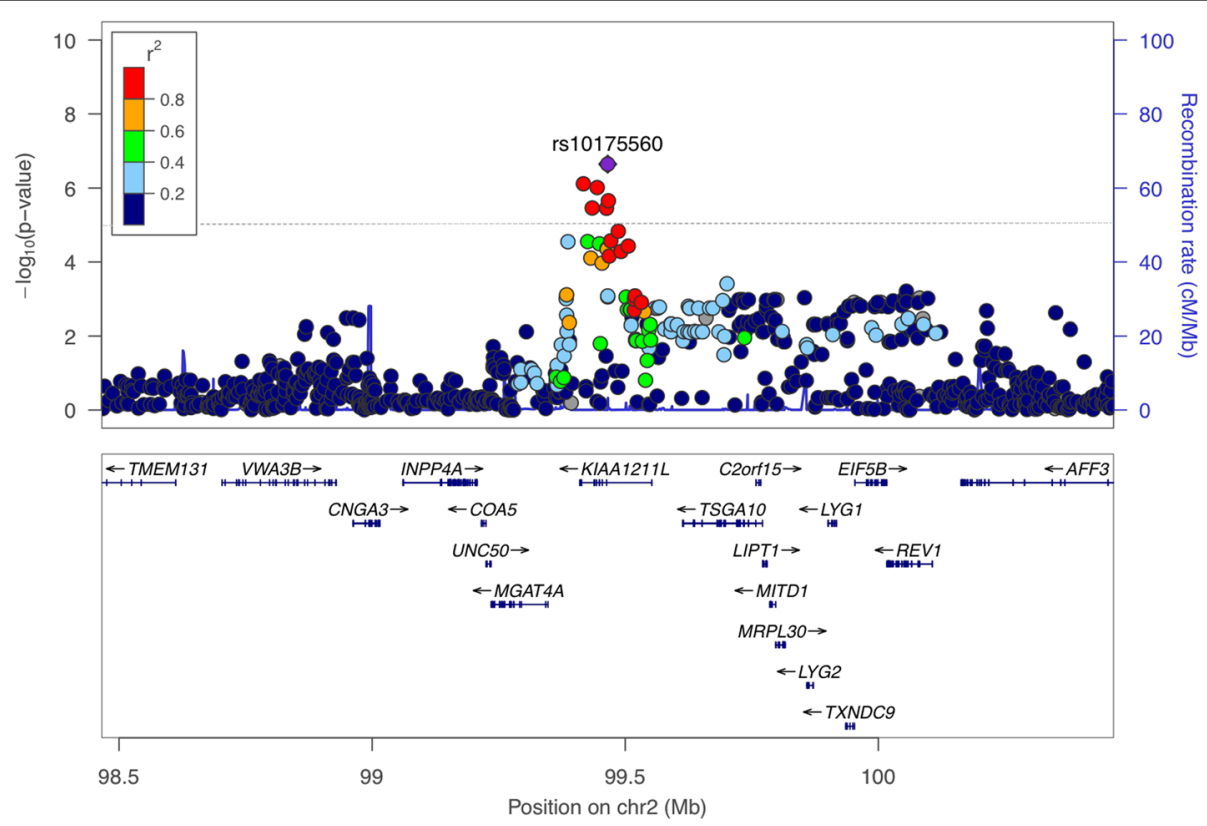

Fig. 2 Regional Manhattan plot of the chromosome 2q11.2 region showing the FaSTLMM analysis of the opioid use disorder patients compared to controls. The LD heat map is based on the hg19/1000genomes NOV/2014 reference set. The SNPs are colour coded according to $r^{2}$ measured based on the pairwise LD with the index SNP rs 10175560 ( $p$-value $=2.24 \times 10^{-7}$ ) shown in purple. Two other SNPs mapped to the KIAA1211L gene clustered in high LD indicating a possible candidate locus for opioid use disorder 


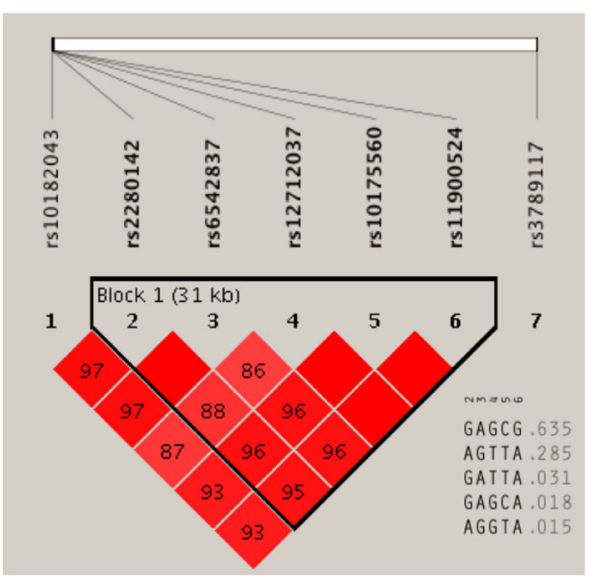

\begin{tabular}{lcccc}
\hline Block 1 & Haplotype Frequency & Case, Control Frequencies & Chi Square & P value \\
\hline GAGCG & 0.635 & $0.535,0.713$ & 30.545 & $\mathbf{3 . 2 6 \times 1 0 ^ { - 8 }}$ \\
\hline AGTTA & 0.285 & $0.369,0.219$ & 24.572 & $\mathbf{7 . 1 6 \times 1 0 ^ { - 7 }}$ \\
\hline GATTA & 0.031 & $0.035,0.028$ & 0.419 & 0.5176 \\
\hline GAGCA & 0.018 & $0.013,0.022$ & 1.079 & 0.2989 \\
\hline AGGTA & 0.015 & $0.025,0.008$ & 4.333 & 0.0374 \\
\hline
\end{tabular}

Fig. 3 The state of the LD between the SNPs located above the GWAS suggestive line $1 \times 10^{-5}$ on chromosome 2, based on opioid use disorder patients compared to controls from the UAE population. Block 1 includes 5 SNPs mapped to the KIAA1211L gene that are significantly associated with opioid use. The numbers in the square represent the percentage of the $r^{2}$ value calculated from the genotype data of the SNPs. The haplotype variations, frequencies and P-values illustrated with the GAGCG $\left(p\right.$-value $\left.=3.26 \times 10^{-8}\right)$ haplotype the most significant

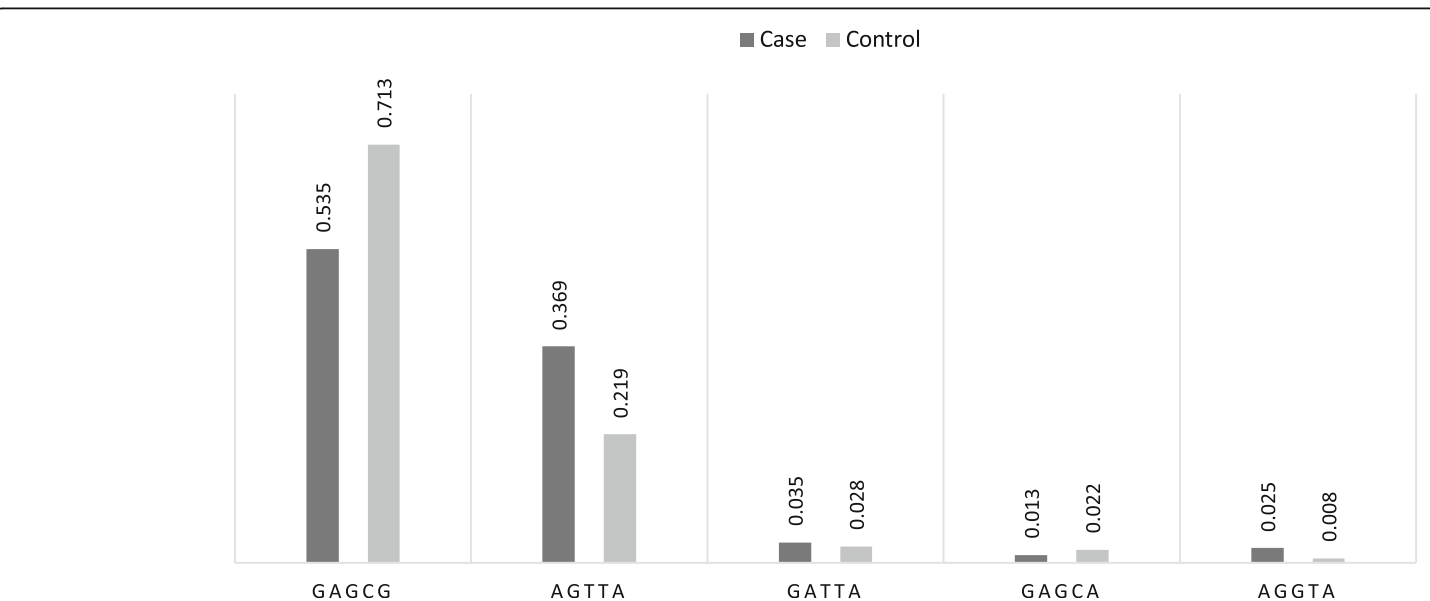

Fig. 4 The distribution of the haplotypes in block 1 that include 5 SNPs between opioid users and controls. Haplotype 1 (GAGCG) the most statistically significant with higher frequency (0.713) in control group. Haplotype 2 (AGTTA) higher in cases (0.369) than control (0.219) 
rs11900524). These 7 SNPs were organized into haplotypes, with block 1 including the five SNPs: rs2280142, rs6542837, rs12712037, rs10175560, and rs11900524. Two haplotypes GAGCG and AGTTA were associated with opioid use disorders at $p$-values of $3.26 \times 10^{-8}$ and $7.16 \times 10^{-7}$, respectively.

Fig. 4 provides a summary of the 5-point haplotype frequencies for block 1 in opioid users and controls. The frequency of haplotype 1 (GAGCG) at 0.713 was higher in the control group relative to the cases. In contrast, the frequency of haplotype 2 (AGTTA) was higher in cases (0.369) than in the control group (0.219).

The analysis was extended to include the chromosome 2 SNPs above the $p$-value $1 \times 10^{-4}$. Linkage Disequilibrium data of 31 SNPs is shown in Additional file 1: Figure. S1. Six haplotype blocks were constructed using these 31 SNPs. Block 1 and 2 mapped to the chromosomal region that coincided with KIAA1211L. Additional file 2: Table S1 also provides a summary of the haplotype distribution, the frequencies of each haplotype, and $p$-value of the cases and control groups.

A number of locations on chromosome 2 was shown to be associated with substance use and other psychiatry disorders. Fig. 5 summaries these association including the KIAA1211L loci identified in this study.

\section{Discussion}

This study is an extension of a GWA study, the first of its kind, involving SUD patients in the UAE population. In the initial GWAS, three SNPs: rs118129027, rs74477937 and rs78707086 were below the GWAS significance level but highly suggestive of an association. These mapped to a region on chromosome 7, which coincides with the YAE1D1 locus. Separately, the function of this gene was discussed as a possible candidate locus for substance use disorder [23]. For this study, the initial GWAS was stratified based on the most common substance of use, which were opioids. These are the most common substance of use in the cohort and included both illicit (e.g. heroin) and prescription medication (e.g. Tramadol). Following data stratification two SNPs: rs118129027 and rs74477937 on chromosome 7 were found to be significantly associated with opioid use disorder. A third SNP rs78707086 did not achieve GWAS significance levels however, it was considered as a potential SNP as it was in strong LD with the top two SNPs (rs118129027 and rs74477937).

Just below the level of significance for GWA studies set at $5.00 \times 10^{-8}$, seven SNPs on chromosome 2 suggested a potential locus that was associated with opioid use disorder (highlighted in Fig. 1) and considered worthy of further analysis. Five of the SNPs mapped to the KIAA1211L gene as illustrated in the regional Manhattan plot of chromosome 2q11.2 (Fig. 2). The SNP rs10175560 was used as the index SNP and was shown to be in high LD with other SNPs mapped to KIAA1211L. This could be a possible candidate locus however, the function of KIAA1211L is not yet known. Nevertheless, an association between KIAA1211L was reported in a study involving bipolar disorder [31]. In a

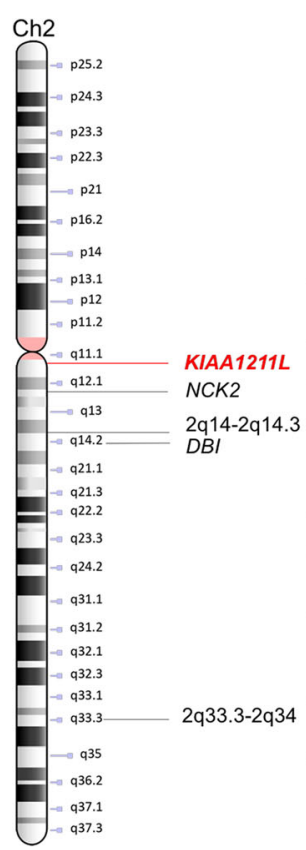

\begin{tabular}{|c|c|c|c|}
\hline Region & Gene & Association & Reference \\
\hline $2 q 11.2$ & KIAA1211L & Opioid Use Disorder & This study \\
\hline $2 q 11.1-2 q 13$ & Include Range of Genes & $\begin{array}{l}\text { Developmental Delay, } \\
\text { Intellectual Disability, } \\
\text { Autism, Congenital } \\
\text { Anomalies and } \\
\text { Psychiatric Disorders. }\end{array}$ & Riely et al, 2015 \\
\hline $2 q 12.2$ & NCK2 & Opioid in African & Liu et al, 2013 \\
\hline $2 q 14.2$ & $\begin{array}{l}\text { DBI (Diazepam Binding } \\
\text { Inhibitor) }\end{array}$ & $\begin{array}{l}\text { Borderline Personality } \\
\text { Disorder }\end{array}$ & Conti et al, 2013 \\
\hline $2 q 14-2 q 14.3$ & Include Range of Genes & $\begin{array}{l}\text { Alcohol Dependence } \\
\text { with Conduct Disorder } \\
\text { and Suicide Attempt }\end{array}$ & Dick et al, 2010 \\
\hline $2 q 33.3-2 q 34$ & Include Range of Genes & Opioid Sensitivity & Nishizawa et al, 2014 \\
\hline
\end{tabular}

Fig. 5 Published associations for chromosome $2 q$ regions from $2 q 11$ to $2 q 34$ and SUD as well as other psychiatric disorders 
study by Castellanie and colleagues (2014) [32] studied copy number variants $(\mathrm{CNVs})$ in six monozygotic twin pairs for differences in schizophrenia. The monozygotic twins discordant (MZD) approach has been successful in identifying rare variants in schizophrenia. The CNV result in, loss (deletion) or gain (duplication/amplification) of a particular segment of the genome. Castellanie et al (2014) [32] reported CNV loss in KIAA1211L in the monozygotic twin pair to be unique in the affected pair with paranoid schizophrenia and not reported in the normal twin pair. This was the first report of this $\mathrm{CNV}$ in the database of genomic variants (DGV). Consequently, this CNV loss suggests that this region carries a potential candidate for schizophrenia. In addition, Hicks et al (2016) [33] investigated molecular markers in children with acute lymphoblastic leukaemia (ALL) Central Nervous System (CNS). The current treatment strategy for ALL is a combined systemic chemotherapy and CNS-directed treatment (cranial radiation, intrathecal methotrexate, or combination). The CNS classification is important in ALL patients in order to determine the efficiency of CNS-directed the therapy. The current classification is based on the presence of blast cells in the cerebrospinal fluid (CSF). It is classified to CNS1 (no detectable blast cells), CNS2 (presence of $<5$ leukocytes per $\mu \mathrm{l}$ with detectable blast) and CNS3 (presence of $>5$ over CNS leukaemia with identifiable blast) [34]. In order to avoid over-treatment or under-treatment in children with ALL, there is a demand for precision assessment of CNS disorders from those without by identifying molecular markers that distinguish each class of CNS. Hicks et al (2016) [33] investigated the differences between gene expression of each level of the CNS in patients. They reported 40 highly significant genes expressed differently between patients with CNS2 and CNS3. The KIAA1211L was identified as one of the sig-

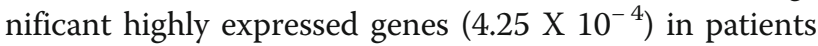
with CNS3 [33]. This can help in determining the intensity of the CNS-directed therapy. Therefore, KIAA1211L can be flagged as distinguishing molecular markers for CNS3 typed patients with ALL. The overall finding highlights the involvement of $K I A A 1211 \mathrm{~L}$ in psychiatric disorders or within the central nervous system and adds weight in support of our results that suggest an association between KIAA1211L and opioid use disorder.

In this study, we also investigated SNPs that were in LD with the index SNP rs10175560. The haplotype compositions and their respective frequencies are illustrated in Fig. 3. In block 1, two haplotypes (GAGCG and AGTTA) were significantly associated with opioid use disorder. For haplotype 1 GAGCG the frequency in controls (0.713) was higher than in the cases (0.535), suggesting a possible protective role to opioid use (Fig. 4). On the other hand, haplotype 2 AGTTA was higher in cases (0.369) when compared to the control group $(0.219)$ that suggest possible involvement of this haplotype in the development of opioid use disorder. The remaining three haplotypes that were characterized (GATTA, GAGCA and AGGTA) were not statistically significant with opioid use (Fig. 4).

By expanding the haplotype analysis to include SNPs on chromosome 2 from the $p$-values from $1 \times 10^{-8}$ to $1 \times 10^{-4}$, six blocks were generated and illustrated in Additional file 1: Figure. S1. The haplotype variations in each block with the classification between cases and controls are summarised in Additional file 2: Table S1. Block 1 and block 2 mapped to the KIAA1211L gene and suggests a potential role in opioid use disorder. In Block 1 , two haplotypes, namely CTAAGT $(p$-value $=$ $\left.5.51 \times 10^{-8}\right)$ and TCTGAC $\left(p\right.$-value $\left.=2.19 \times 10^{-7}\right)$ were significantly associated with opioid use. The 2 SNP block 3 mapped to the ACOXL (acyl-CoA oxidese like) gene with the GA combination $\left(p\right.$-value $\left.=7.69 \times 10^{-7}\right)$ shown to be the most significant haplotype. Blocks 4 and 5 mapped to the $V W C 2 L$ gene (von Willebrand factor $\mathrm{C}$ domain containing protein 2 like) and block 6 mapped to OR6B3 (olfactory receptor family 6 subfamily B member 3). There were no direct associations found in the literature between these genes and SUD or specifically opioids. Nevertheless, further investigation would be required to understand the associations reported here and possible mechanisms that may link these genes to opioid use.

Various studies [3, 18, 35-37] have discussed the importance of the q-arm region on chromosome 2 in SUD and other psychiatric disorders (Fig. 5). The NCK2 gene is located on the long arm of chromosome 2 (2q12.2) is located proximally to the region of the KIAA1211L locus identified in this study. Liu et al (2012) [18] reported a significant association between a SNP (rs2377339) on NCK2 $\left(p\right.$-value $\left.=3.12 \times 10^{-8}\right)$ with opioid use disorder in a population of African descent. The NCK family is classified as a group of adaptor proteins that interact with other proteins. Specifically, it is involved in the regulation of receptor protein tyrosine kinase signalling and the regulation of actin cytoskeleton and cell movement [32]. These findings suggest evidence for the involvement of the NCK2 in the pathway of opioid use disorder, that highlight the potential role of the KIAA1211L locus in the disorder.

Another genetic region in close proximity to KIAA1211L contains the Diazepam Binding Inhibitor $(D B I)$. The $D B I$ loci on chromosome 2q.14.2 encodes for a protein that has been suggested to be involved in the regulation of number of functions in the CNS, including responses to stress, depression, anxiety and neuropsychiatric disorder [35]. Based on the chromosomal position and the proximity of the $D B I$ to the KIAA1211L, the 
possible involvement of the KIAA1211L in the CNS should be explored.

Another region of interest on chromosome 2 was mapped to a region bound by $2 \mathrm{p} 14$ and $2 \mathrm{q} 14.3$. This region has been studied in the context of various behavioural conditions, alcohol dependence, suicide attempts and conduct disorder have been implicated [36]. Dick et al (2010) [36] provided evidence for the involvement of 23 genes (NTSR2, TRIB2, PPM1G, MEMO1, HAAO, MTIF2, CCDC139, EHBP1, BUB1, TTL, CKAP2L, MGAT5, ARHGAP15, KIAA1189, COBLLI, FAM130A2, LPR2, CHN1, PRKRA, PPP1R1C, LOC402117, IL8RA, FARP2) on chromosome 2 in alcohol dependence. These genes potentially contribute to alcohol dependence, conduct disorder and/or suicide attempts. Other regions across chromosome 2 have been significantly associated with a range of psychiatric disorders. Riley et al (2015) [37] reported genomic imbalance for the region between $2 \mathrm{q} 11.2$ and $2 \mathrm{q} 13$ in patients with selected clinical symptoms including developmental delay, intellectual disability and congenital anomalies. A deletion in the region from $2 \mathrm{q} 12.2$ to $2 \mathrm{q} 13$ has been reported in patients with developmental delay and dysmorphic features [38]. Furthermore, a deletion in the 2 q21 region was reported in patients with developmental delay/intellectual disabilities, attention deficit hyperactivity disorder, epilepsy and other neurobehavioral abnormalities [39]. The overlap of the regions between studies implicate gene(s) with the CNS that could potential contribute to the disorder.

\section{Conclusion}

Overall, findings in this study propose KIAA1211L as a putative candidate locus associated with opioid use disorder. Even though, the function is not fully understood, the region contains genes that are involved in the expression and function of the CNS proteins. In addition, the evidence presented here supports the role of this region in psychiatric disorders, including SUD. Future studies should consider replication of the KIAA1211L locus in a larger cohort, including groups of individuals of Arabian descent. In addition, more research is required to investigate the role of KIAA1211L in opioid use disorder and other substances. Further assessment of haplotype variations, specifically with reference to opioid use is required in order to understand their roles in opioid use disorder. Future research should consider in depth sequencing of the KIAA1211L gene and the surrounding region, comparing variants in opioid use patients and controls to reveal any further genetic associations in order to contribute to the understanding of underlining mechanisms that result in the disorder.

\section{Supplementary information}

Supplementary information accompanies this paper at https://doi.org/10. 1186/s12888-019-2425-8.

Additional file 1 Figure. S1. The state of the LD of the SNPS located between the GWAS line and suggestive line $\left(1 \times 10^{-8}-1 \times 10^{-4}\right)$ on chromosome 2, based on opioid use disorder patients compared to controls from the UAE population. Block 1, 2 mapped to the KIAA1211L gene and is significantly associated with opioid use disorder in this cohort.

Additional file $\mathbf{2}$ Table S1. The distribution of haplotype association between the GWAS line and suggestive line $\left(1 \times 10^{-8}-1 \times 10^{-4}\right)$ on chromosome 2 based on opioid users' patients compared to controls from the UAE population.

\section{Abbreviations}

ALL: Acute Lymphoblastic Leukaemia; CNS: Central Nervous System;

DBI: Diazepam Binding Inhibitor; DSM-5: Diagnostic and Statistical Manual_5;

EFR: Emirates Family Registry; GWAS: Genome Wide Association Study;

HWE: Hardy Weinberg Equilibrium; IBD: Identity by Descent; MAF: Minor

Allele Frequency; MDS: Multi-dimensional Scaling; NRC: National

Rehabilitation Centre; QC: Quality Control; QQ: Quantile-Quantile;

SUD: Substance Use Disorder; UAE: United Arab Emirates

\section{Acknowledgments}

We acknowledge the support and assistance from staff at the UAE National Rehabilitation Center (NRC) in their assistance in samples and information collections. We extend our gratitude to staff at the Center of Biotechnology at Khalifa University of Science and Technology, Abu Dhabi, United Arab Emirates for their assistance in the laboratory.

\section{Authors' contributions}

The first author (Hiba Alblooshi) was involved in the design of the study, was directly involved in the laboratory experiments, data analysis and preparation of this manuscript. Habiba Al Safar, Gary Hulse and Guan Tay are supervisors of Hiba Alblooshi, who is a doctoral student at the University of Western Australia. They assist by providing input on all elements of this study from design to reporting. The NRC team, comprising Ahmed El Kashef, Mansour Shawky and Hamad Al Ghaferi, were consulted during the study design phase. The team also provided clinical assessment and were involved in recruiting the patients and providing access to data on the patients. All authors approved the content of this manuscript.

\section{Funding}

This research was funded by the UAE National Rehabilition Center (NRC), Abu Dhabi, UAE. The NRC team were involved in the study design, clinical assessment, patient's recruitment and manuscript reviewing.

\section{Availibility of data and materials}

The datasets used and analysed during the current study are available from the corresponding author on reasonable request.

\section{Ethics approval and consent to participate}

The ethical approvals were obtained from the UAE National Rehabilitation Center (NRC) in Abu Dhabi, UAE. Co-approval was obtained from the Human Research Ethics Committee of the University of Western Australia (UAW) (RA) 4/1/6715). Written informed consent was obtained from each participant.

\section{Consent for publication}

All particpants provided their consent for their de-identified data to be published.

\section{Competing interets}

The authors declare they have no competing of intrests.

\section{Author details}

'Division of Psychiatry, the University of Western Australia, Crawley, Western Australia, Australia. ${ }^{2}$ School of Human Science, The University of Western Australia, Crawley, Western Australia, Australia. ${ }^{3}$ College of Medicine and Health Science, The United Arab Emirates University, Al Ain, United Arab 
Emirates. ${ }^{4}$ Center of Biotechnology, Khalifa University of Science and Technology, Abu Dhabi, United Arab Emirates. ${ }^{5}$ Department of Biomedical Engineering, Khalifa University of Science and Technology, Abu Dhabi, United Arab Emirates. ${ }^{6}$ National Rehabilitation Center, Abu Dhabi, United Arab Emirates. 'School of Health and Medical Science, Edith Cowan University, Joondalup, Western Australia, Australia.

\section{Received: 13 February 2019 Accepted: 30 December 2019}

\section{Published online: 31 January 2020}

\section{References}

1. Degenhardt L, Charlson F, Mathers B, Hall WD, Flaxman AD, Johns N, et al. The global epidemiology and burden of opioid dependence: results from the global burden of disease 2010 study. Addiction. 2014;109(8):1320-33.

2. Substance Abuse and Mental Health Services Administration, Results from the 2012 National Survey on Drug Use and Health: Mental Health Findings, NSDUH Series H-47, HHS Publication No. (SMA) 13-4805. Rockville, MD:

Substance Abuse and Mental Health Services Administration; 2013.

3. Nishizawa D, Fukuda K, Kasai S, Hasegawa J, Aoki Y, Nishi A, et al. Genomewide association study identifies a potent locus associated with human opioid sensitivity. Mol Psychiatry. 2014;19(1):55-62.

4. Van Amsterdam J, Phillips L, Henderson G, Bell J, Bowden-Jones O, Hammersley $R$, et al. Ranking the harm of non-medically used prescription opioids in the UK. Regul Toxicol Pharmacol. 2015;73(3):999-1004.

5. Manchikanti L, Helm S, Fellows B, Janata JW, Pampati V, Grider JS, et al. Opioid epidemic in the United States. Pain Physician. 2012;15(3 Suppl):ES9-38.

6. Holmes D. Prescription drug addiction: the treatment challenge. Lancet. 2012;379(9810):17-8

7. International Narcotics Control Board. Narcotic drugs technical report: estimated world requirements for 2012-statistics for 2010. New York: United Nations; 2012.

8. Ruscitto A, Smith BH, Guthrie B. Changes in opioid and other analgesic use 1995-2010: repeated cross-sectional analysis of dispensed prescribing for a large geographical population in Scotland. Eur J Pain. 2015;19(1):59-66.

9. Zin CS, Chen LC, Knaggs RD. Changes in trends and pattern of strong opioid prescribing in primary care. Eur J Pain. 2014;18(9):1343-51.

10. Dale-Perera A, Goulao J, Stover H. Quality of care provided to patients receiving opioid maintenance treatment in Europe: results from the EQUATOR analysis. Heroin Addiction Relat Clin Probl. 2012;14:23-38.

11. Elkashef A, Zoubeidi T, Thomas RA, Al Hashmi H, Lee AJ, Aw T-C ea. A profile of patients with substance use disorders and treatment outcomes: A 10-year retrospective study from the National Rehabilitation Center 2013. Int J Prev Treat Subst Use Dis. 2013:62-75.

12. Alblooshi H, Hulse GK, El Kashef A, Al Hashmi H, Shawky M, Al Ghaferi H, et al. The pattern of substance use disorder in the United Arab Emirates in 2015: results of a National Rehabilitation Centre cohort study. Subst Abuse Treat Prev Policy. 2016;11(1):19.

13. Al Ghaferi H, Yousif Ali A, Gawad T, Wanigaratne S. Developing substance misuse services in United Arab Emirates: the National Rehabilitation Centre experience. BJPsych International. 2017;14:92-6.

14. Bierut L. Genetic vulnerability and susceptibility to substance dependence. Neuron. 2011;69(4):618-27.

15. Kendler KS, Jacobson KC, Prescott CA, Neale MC. Specificity of genetic and environmental risk factors for use and Abuse/dependence of Cannabis, cocaine, hallucinogens, sedatives, stimulants, and opiates in male twins. Am J Psychiatr. 2003;160(4):687-95.

16. Van den Bree M, MB JEO, Neale MC, Pickens RW. Genetic and environmental influences on drug use and abuse/dependence in male and female twins. Drug Alcohol Depend. 1998:52(3):231-41.

17. Gelernter J, Gelernter H, Kranzler R, Sherva R, Koesterer L, Almasy H, et al. Genome-wide association study of opioid dependence: multiple associations mapped to calcium and potassium pathways. Biol Psychiatry. 2014;76(1):66-74

18. Liu Z, Guo X, Jiang Y, Zhang H. NCK2 is significantly associated with opiates addiction in African-origin men. Sci World J. 2013;2013:748979.

19. Nielsen DA, Ji F, Yuferov V, Ho A, He C, Ott J, et al. Genome-wide association study identifies genes that may contribute to risk for developing heroin addiction. Psychiatr Genet. 2010;20(5):207-14.

20. Nelson EC, Lynskey MT, Heath AC, et al. Ankk1, ttc12, and ncam1 polymorphisms and heroin dependence: importance of considering drug exposure. JAMA psychiatry. 2013;70(3):325-33.
21. Nelson EC, Agrawal A, Heath AC, Bogdan R, Sherva R, Zhang B, et al. Evidence of $\mathrm{CNIH} 3$ involvement in opioid dependence. Mol Psychiatry. 2016;21(5):608-14

22. Li D, Zhao H, Kranzler HR, Li MD, Jensen KP, Zayats T, et al. Genome-wide association study of copy number variations (CNVs) with opioid dependence. Neuropsychopharmacology: official publication of the American College of Neuropsychopharmacology. 2015;40(4):1016-26.

23. Alblooshi H, Al Safar H, Fisher HF, Cordell HJ, El Kashef A, Al Ghaferi H, et al. A case-control genome wide association study of substance use disorder (SUD) identifies novel variants on chromosome 7p14.1 in patients from the United Arab Emirates (UAE). Am J Med Genet B Neuropsychiatr Genet: the official publication of the International Society of Psychiatric Genetics. 2019; 180(1):68-79.

24. Alsafar H, Jama-Alol K, Hassoun AK, Tay G. The prevalence of type 2 diabetes mellitus in the United Arab Emirates: justification for the establishment of the emirates family registry. Int J Diabetes Dev Ctries. 2012 32(1):25-32.

25. World Medical Association Declaration of Helsinki. Recommendations guiding physicians in biomedical research involving human subjects. JAMA. 1997:277(11):925-6.

26. Purcell SNB, Todd-Brown K, Thomas L, Ferreira MAR, Bender D, Maller J, et al. PLINK: a toolset for whole-genome association and population-based linkage analysis. Am J Hum Genet. 2007;81:559-75

27. Lippert C, Listgarten J, Liu Y, Kadie CM, Davidson RI, Heckerman D. FaST linear mixed models for genome-wide association studies. Nat Methods. 2011:8(10):833-5.

28. Fadista J, Manning AK, Florez JC, Groop L. The (in) famous GWAS P-value threshold revisited and updated for low-frequency variants. Eur J Hum Genet. 2016;24(8):1202-5.

29. Pruim RJ, Welch RP, Sanna S, Teslovich TM, Chines PS, Gliedt TP, et al. LocusZoom: regional visualization of genome-wide association scan results. Bioinform. 2010;26(18):2336-7.

30. Barrett JC, Fry B, Maller J, Daly MJ. Haploview: analysis and visualization of LD and haplotype maps. Bioinform. 2005;21(2):263-5.

31. Scott LJ, Muglia P, Kong XQ, Guan W, Flickinger M, Upmanyu R, et al. Genome-wide association and meta-analysis of bipolar disorder in individuals of European ancestry. Proc Natl Acad Sci U S A. 2009;106(18): 7501-6.

32. Castellani CA, Awamleh Z, Melka MG, O'Reilly RL, Singh SM. Copy number variation distribution in six monozygotic twin pairs discordant for schizophrenia. Twin Res Hum Genet. 2014;17(2):108-20.

33. Hicks C, Sitthi-Amorn J, Douglas J, Ramani R, Miele L, Vijayakumar V, et al. Molecular analysis of central nervous system disease spectrum in childhood acute lymphoblastic leukemia. Clin Med Insights Oncol. 2016;10:5-15.

34. Pui C-H, Howard SC. Current management and challenges of malignant disease in the CNS in paediatric leukaemia. Lancet Oncol. 2008;9(3):257-68.

35. Conti E, Nacinovich R, Bomba M, Uccellini O, Rossi MS, Casati M, et al. Diazepam binding inhibitor and dehydroepiandrosterone sulphate plasma levels in borderline personality disorder adolescents. Neuropsychobiology. 2014:69(1):19-24.

36. Dick DM, Meyers J, Aliev F, Nurnberger J, Kramer J, Kuperman S, et al. Evidence for genes on chromosome 2 contributing to alcohol dependence with conduct disorder and suicide attempts. Am J Med Genet B Neuropsychiatr Genet. 2010;153B(6):1179-88.

37. Riley KN, Catalano LM, Bernat JA, Adams SD, Martin DM, Lalani SR, et al. Recurrent deletions and duplications of chromosome $2 q 11.2$ and $2 q 13$ are associated with variable outcomes. Am J Med Genet A. 2015;167A(11):2664-73.

38. Dittwald P, Gambin T, Szafranski P, Li J, Amato S, Divon MY, et al. NAHRmediated copy-number variants in a clinical population: mechanistic insights into both genomic disorders and Mendelizing traits. Genome Res. 2013:23(9):1395-409.

39. Dharmadhikari AV, Kang S-HL, Szafranski P, Person RE, Sampath S, Prakash SK, et al. Small rare recurrent deletions and reciprocal duplications in 2q21.1, including brain-specific ARHGEF4 and GPR148. Hum Mol Genet. 2012;21(15): 3345-55.

\section{Publisher's Note}

Springer Nature remains neutral with regard to jurisdictional claims in published maps and institutional affiliations. 